\title{
Clinical ethics report on the resuscitation of a patient in the emergency department with an uncertain resuscitation status and an implantable cardiac defibrillator
}

\author{
Gregory Neal-Smith $\odot,{ }^{1}$ Adam Crellin, ${ }^{2}$ Rebekah Caseley ${ }^{1}$
}

\begin{abstract}
${ }^{1} A \& E$, Oxford University Hospitals, Oxford, UK ${ }^{2}$ Medical Sciences Division, Oxford University Medical School, Oxford, UK
\end{abstract}

\section{Correspondence to}

Mr Gregory Neal-Smith, John Radcliffe Hospital, Oxford OX3 9DU, UK:

gregnealsmith@doctors.org.uk

Received 18 November 2019

Revised 11 March 2020

Accepted 20 March 2020

Published Online First

6 April 2020
Check for updates

(C) Author(s) (or their employer(s)) 2020. No commercial re-use. See rights and permissions. Published by BMJ.

To cite: Neal-Smith $\mathrm{G}$, Crellin A, Caseley R.

$J$ Med Ethics

2020:46:581-583.

\section{SUMMARY}

Cardiopulmonary resuscitation of a patient with an uncertain resuscitation status, and a discharging implantable cardiac defibrillator, presents a significant ethical challenge to healthcare professionals in the emergency department. Presently, no literature discusses these challenges or their implications for ethical healthcare delivery. This report will discuss the issues that arose during the management of such a case and attempt to raise awareness among healthcare professionals to ensure better preparation for similar situations.

\section{INTRODUCTION}

An 82-year-old man with an implantable cardiac defibrillator (ICD) was brought to the emergency department (ED) following an episode of syncope. He subsequently had a cardiac arrest, and as no DNACPR ("Do not attempt cardiopulmonary resuscitation") decision was documented resuscitation efforts were started. The difficulty caring for the patient was threefold: an unclear DNACPR status, a rapidly fluctuating clinical condition due to a discharging ICD and a lack of consultant presence resulting in an ethically challenging resuscitation scenario.

Clear documentation of DNACPR decisions is extremely important and it should be easily accessible to avoid confusion during an emergency. As a result of this case, we would advocate a patient's DNACPR status is displayed on electronic patient records, if used within Trusts. Furthermore, immediate completion of DNACPR forms should occur whenever patients express this wish, regardless of location, including specialty clinics. We would also stress the importance of pre-emptive discussions with patients and family, and consultant availability.

\section{THE CASE}

An 82-year-old man was brought to the ED by ambulance in the evening with his wife and daughter, following an episode of syncope.

He had a background of an ICD inserted 18 months earlier and subsequent cardiac resynchronisation therapy, atrial fibrillation, recurrent ventricular tachycardia (VT), coronary artery bypass graft, severe left ventricular systolic impairment, hypertension and chronic kidney disease. He was admitted under cardiology the previous month for ablation due to worsening heart failure symptoms and recurrent episodes of slow VT.

He lived with his wife at home and was fully independent. There was no available documentation of the patient's resuscitation status, and as the patient appeared well this was not discussed during his initial assessment.

The patient attended the ED as he had felt dizzy and fallen over, hitting his head on the bathroom sink. He had amnesia of the event and there was a presumed loss of consciousness. During the same day the patient had multiple episodes of presyncope, during which he noted that he could not feel his radial pulse. He sent an interrogation of his ICD to the technicians which was reported as normal.

In the ED, his examination was unremarkable and his observations were stable. His ECG showed a paced rhythm with a heart rate of 63 . His blood and a CT head (completed in accordance with NICE guidelines on head injury) were normal.

The patient was diagnosed with likely cardiac syncope and was admitted under the medical team for interrogation of his ICD in the morning. An hour later, the patient collapsed while changing for bed. The patient lost consciousness and had no palpable central pulse, and his monitor showed ventricular fibrillation (VF). A resus call was put out, cardiopulmonary resuscitation was started and his ICD delivered several shocks in an attempt to cardiovert his VF.

The patient alternated between VT, VF and sinus rhythm. During this treatment, his wife stated that at his last cardiology clinic appointment he had informed his cardiologist that he did not want to be resuscitated. He had also handed in a letter detailing this. The cardiologist had not signed a DNACPR form, so his decision had not been formalised.

As the patient had a witnessed cardiac arrest, he was in a shockable rhythm and there was no formal DNACPR, some members of the team were uncomfortable about whether CPR should be stopped. Following a discussion with the team and family, it was felt that the patient was unlikely to recover due to his refractory VF and poor cardiac function. In view of the patient's previously expressed wishes regarding resuscitation, the decision was made to deactivate his ICD. Shortly after this, the patient sadly died. 


\section{ETHICAL REVIEW}

The implementation of cardiovascular implanted electronic devices, including ICDs, has dramatically increased in recent years due an ageing population and an increased number of indications for use. ${ }^{1}$ At present, extensive literature exists discussing the deactivation of ICDs during end-of-life care and the resuscitation of patients with an uncertain resuscitation status. However, to our knowledge, no literature currently discusses the legal and ethical challenges of resuscitating a patient with an uncertain resuscitation status and an active ICD in situ. Therefore, this case report will discuss the issues which arose during the management of such a case, to broaden the discussion so those facing similar challenges may be better prepared.

The difficulty caring for our patient involved ethical and logistical issues over three areas. First, his resuscitation status was unclear due to the lack of documentation; second, the patient had a complex resuscitation which prevented the team from focusing on the ethical issue of continuing CPR and third, the duty ED consultant was not present to assist in the decision.

With regards to his resuscitation status, the law supplies clear guidance to ensure appropriate action can be taken. Common law states that a person with capacity can refuse treatment, including CPR, but that a person may not demand treatment that a doctor does not consider to be in their best interests. ${ }^{2}$ If the person lacks capacity, such as in this case when the patient arrested, then the Mental Capacity Act $2005^{3}$ applies. It states that decisions should be made in the patient's best interests, taking into account his or her wishes before capacity was lost and those of the people close to the patient. ${ }^{3}$ The Mental Capacity Act 2005 also encompasses a patient's right to advance refusal of treatment, including CPR, should they lose capacity. ${ }^{3}$ The final piece of legislation was established in the case of Airedale NHS Trust vs Bland. It clarified that withholding or withdrawing life-sustaining treatment may be appropriate if it is not in the patient's best interest. ${ }^{4}$

Considering the three pieces of legislation in the context of our case, it is our duty to provide care in the best interests of the patient as no formal documentation of resuscitation status was available. However, determining a patient's best interests is not always clear. Little clinical information about the patient is often available in an emergency scenario, thus the only information the resuscitation team had was the views of the family. In the absence of other evidence, it seems reasonable to believe that the family has the patient's best interests at heart and to cease resuscitation. However, in our case it was only after the team agreed treatment was futile, that resuscitation was stopped. We would therefore encourage clinicians to discuss resuscitation status with patients at risk of cardiac arrest in the ED prior to deterioration. Also it is crucial to involve the family in this first step if consent is given, and if a patient has deteriorated, to urgently discuss families' views to establish the patient's prior views on resuscitation.

The second challenging aspect was a logistical problem as the patient rapidly fluctuated between the shockable and nonshockable side of the advanced life support algorithm, with intermittent periods of spontaneous circulation. He received airway management, amiodarone and electrolyte correction throughout.

This meant that there was limited opportunity for the team to focus on the ethical question of whether to continue resuscitation or stop in accordance with the presumed wishes of the patient.

The final difficulty was the challenge to manage the treatment of this patient without consultant input, particularly with the emerging information about the patient's prior wishes. Studies investigating differences in ethical decision-making between consultants and non-consultant doctors in the ED appear to be non-existent at the time of writing. Also, studies investigating differences in patient outcomes in the ED between consultants and non-consultant doctors in the UK are few. However, a 5 -year retrospective study of patient survival following trauma, compared with predicted survival according to the Trauma Score-Injury Severity Score, showed that patients treated by consultants had significantly better survival than those treated by non-consultant grade doctors. ${ }^{5}$ A study analysing the impact of senior review of initial assessments made by junior doctors in the ED reported that senior doctors changed the primary outcome plan in $28 \%$ of cases. ${ }^{6}$

Overall, numerous reviews conclude that a delay in consultant involvement in patient care increases mortality and morbidity in a range of fields, including emergency medicine. ${ }^{78}$ This is thought in part to be due to a consultant's experience which enables them to acknowledge the unusual, unexpected and unfamiliar which in the emergency medical setting can lead to dramatic differences in outcome. ${ }^{8}$ The Royal College of Emergency Medicine recognises this in both their 2010 and 2018 emergency medicine consultants' workforce recommendations, particularly in leading the resuscitation of critically ill patients. ${ }^{910}$ However, a 2012 national audit of patients undergoing CPR as a result of an in-hospital cardiorespiratory arrest demonstrates a very low level of consultant involvement. ${ }^{11}$ They found that only $9 \%$ of cardiac arrest calls were led by a consultant and only $40 \%$ of acute admissions were identified to have been seen by a consultant within 12 hours. ${ }^{11}$ These studies are however difficult to generalise to the whole of the UK. Regarding our case, extrapolation of the above conclusions in the absence of specific evidence regarding ethical decision-making between consultants and non-consultant doctors in the ED indicates a trend towards better decision making when a consultant leads care.

While attempts to resuscitate our patient were rapidly deemed futile and thus a consultant's presence would likely have made little difference to their outcome, the evidence suggests that the experience of a consultant improves the speed of decisionmaking. Therefore a consultant's presence may have enabled the decision regarding resuscitation status to be resolved quicker amidst uncertainty and therefore be in a patient's best interest.

In summary, our patient was difficult to care for because of an uncertain resuscitation status and a discharging ICD in situ, rapid fluctuation in clinical condition and the absence of an experienced consultant. Clear documentation of DNACPR decisions are extremely important and should be easily accessible to avoid the confusion during an emergency. Oxford University Hospitals NHS Trust now displays resuscitation status next to the patient's name on their electronic notes. This should prevent confusion in the future, providing resuscitation decisions are correctly documented. We would advocate the same for all other trusts, as it would aid in similar situations and whenever patients transfer from ward to ward, when information can be omitted from paper notes and during handover. From this case we would stress the importance of discussing DNACPR decisions whenever the opportunity arises, including specialty clinics, and it should not be left to the patient's general practitioner or ED doctor to have that conversation. This would prevent similar situations occurring again. The advanced care of the patient is everyone's responsibility and as the patient told his cardiologist that he did not want to be resuscitated and handed in a letter to this nature, the necessary documents should have been completed in the clinic. In the absence of a clear resuscitation status during cardiac arrest, it is extremely important to consider the family members 
views in order to gauge the patient's best interests. We would also advocate that a consultant grade doctor always be available for cases transferred into the resuscitation room to allow speedy assessment and treatment, particularly in those unexpectedly transferred.

Twitter Gregory Neal-Smith @GregNealSmith1

Contributors All three authors were involved in the clinical case. All fulfilled ICMJE Recommendations 2018 for authorship. GN-S is the corresponding author for the article.

Funding The authors have not declared a specific grant for this research from any funding agency in the public, commercial or not-for-profit sectors.

Competing interests None declared.

Patient consent for publication Next of kin consent obtained.

Provenance and peer review Not commissioned; externally peer reviewed.

ORCID iD

Gregory Neal-Smith http://orcid.org/0000-0002-7907-4277

\section{REFERENCES}

1 Stoevelaar R, Brinkman-Stoppelenburg A, Bhagwandien RE, et al. The incidence and impact of implantable cardioverter defibrillator shocks in the last phase of life: An integrated review, European Journal of Cardiovascular Nursing 2018;17(no. 6):477-85.

2 Burke R (on the application of). $v$ General Medical Council \& Ors, 2005.

3 Britain G. Mental capacity act 2005. London: The Stationery Office, 2005.

4 Airedale NHS trust V Bland 1993.

5 Wyatt JP, Henry J, Beard D. The association between seniority of accident and emergency doctor and outcome following trauma. Injury 1999;30(3):165-8.

6 White A, Armstrong P, Thakore S. Impact of senior clinical review on patient disposition from the emergency department, Emergency Medicine Journal 2010;27(no. 4):262-5.

7 NCEPOD. Emergency admissions: a journey in the right direction? NCEPOD: London, 2007.

8 AOMRC. The benefits of Consultant-Delivered care. London: AOMRC, 2012.

9 The College of Emergency Medicine. "Emergency Medicine Consultants Workforce Recommendations". London: The College of Emergency Medicine, 2010.

10 RCEM. Consultant staffing in emergency departments in the UK. London: The Royal College of Emergency Medicine, 2018

11 NCEPOD. Time to intervene? A reviews of patients who underwent cardiopulmonary resuscitation as a result of an in-hospital cardiorespiratory arrest. London: NCEPOD, 2012 